\title{
Stochastic differential equations driven by fractional Brownian motion with locally Lipschitz drift and their implicit Euler approximation
}

\author{
Shao-Qin Zhang* and Chenggui Yuan ${ }^{\dagger}$ \\ ${ }^{*}$ School of Statistics and Mathematics \\ Central University of Finance and Economics, Beijing 100081, China. \\ Email: zhangsq@cufe.edu.cn \\ ${ }^{\dagger}$ Department of Mathematics, Swansea University, Bay Campus SA1 8EN, UK. \\ Email: C.Yuan@swansea.ac.uk
}

\begin{abstract}
In this paper, a class of one-dimensional stochastic differential equations driven by fractional Brownian motion with Hurst parameter $H>\frac{1}{2}$ is studied. The drift term of the equation is locally Lipschitz and unbounded in the neighborhood of the origin. The existence, uniqueness and positivity of the solutions are proved. We estimate moments including the negative power moments. We also develop the implicit Euler scheme, proved that the scheme is positivity preserving and strong convergent, and obtain rate of convergence. Furthermore, we show that our results can be applied to stochastic interest rate models such as meanreverting stochastic volatility model and strongly nonlinear Aït-Sahalia type model by using Lamperti transformation.
\end{abstract}

AMS Subject Classification (2010): 60H35; 60H10

Keywords: locally Lipschitz drift; fractional Brownian motion; implicit Euler scheme; optimal strong convergence rate; interest rate models 


\section{Introduction}

In this paper, we shall consider a one-dimensional stochastic differential equation (in short SDE) driven by fractional Brownian motion:

$$
\mathrm{d} X_{t}=b\left(t, X_{t}\right) \mathrm{d} t+\sigma \mathrm{d} B_{t}^{H}, X_{0} \geq 0
$$

where $B_{t}^{H}$ is a fractional Brownian motion (fBM for short) with Hurst parameter $H \in(1 / 2,1)$ and the drift term $b(t, x)$ is only local Lipschitz in $x \in(0, \infty)$ and unbounded in the neighborhood of 0 .

There are many stochastic interest models which are characterized by stochastic differential equations driven by Brownian motion. For example: the well known Cox-Ingersoll-Ross (C-I-R for short) model [9] and Ait-Sahalia model [1]. However, in order to capture the real world more precisely, due to the memory effects of fractional Brownian motion, it would be reasonable to replace Brownian motion by $\mathrm{fBM}$ if there are inert investors in this market, see for instance [24]. Additionally, motivated by studying the fractional C-I-R model, a singular fractional SDEs has been discussed in [18] under some conditions. Recently, a general fractional C-I-R with Hurst parameter $H \in(0,1)$ was introduced in [21]. However, some models cannot be covered by the conditions introduced recently in [18], see e.g. the equation (3) in [21] or Example 4.1 and Example 4.2 below. Hence one aim of the present paper is to give more general conditions to cover more stochastic interest rate models by using the Lamperti transformation, even if their coefficients have super-linear growth, see e.g. Example 4.2 for the Ait-Sahalia-type interest rate model for details.

Numerical approximations of SDEs arising from finance are of great interest. For instance, the strong approximation of C-I-R model based on the Euler-type method was shown in [11] and optimal convergence rate was obtained; the strong convergence of Euler-Maruyama (EM) type approximations for Aït-Sahalia type model was given [25]; in [19], the EM approximations for a general mean-reverting stochastic volatility model under regime-switching was presented. For the EM scheme of SDEs with non-Lipschitz coefficients, one can see $[11,25,5,13]$ and references therein. However, the numerical issues for SDEs driven by fBM have not been well studied, comparing with SDEs driven by Brownian motion. Recently, the authors in [15] obtained optimal strong convergence rate of backward Euler scheme for C-I-R model driven by fBM. For more details on numerical scheme for fractional SDEs, we refer reader to $[15,16,17,22]$ and references therein. In the present paper, after a general discussion on (1.1), we investigate the numerical approximation of the solution to this equation when $X_{0}$ is positive. The strong convergence of the numerical scheme is obtained. Based on the Lamperti transformation used as in $[11,15,18]$, our results can cover more interesting models in mathematical finance, such as mean-reverting stochastic volatility model (Example 4.1):

$$
\mathrm{d} Z_{t}=\left(a_{1}-a_{2} Z_{t}\right) \mathrm{d} t+\sigma Z_{t}^{\gamma} \mathrm{d} B_{t}^{H}, Z_{0}>0,
$$


where $\gamma \in[1 / 2,1)$; and Aï-Sahalia type model (Example 4.2):

$$
\mathrm{d} Z_{t}=\left(a_{-1} Z_{t}^{-1}-a_{0}+a_{1} Z_{t}-a_{2} Z_{t}^{r}\right) \mathrm{d} t+\sigma Z_{t}^{\rho} \mathrm{d} B_{t}^{H}, Z_{0}>0,
$$

where $\rho>1, r+1>2 \rho$ and $r \geq 2 \wedge \rho+1$. The stochastic integral in these two models is in the sense of pathwise Riemann-Stieltjes integral developed by Zähle in [27]. Replacing fBM by Brownian motion in equations (1.2) and (1.3), the first model was studied in [19] under regime-switching, and the convergence rate is obtained, the second model was studied in [25], where the convergence rate is not clear. Following the study in $[11,15]$, the positivity preserving implicit Euler-type method is adopted in our paper. Here, not only is the strong convergence shown, but also the convergence rate is obtained. For concrete examples presented above, the convergence order of the mean-reverting stochastic volatility model is the Hurst parameter $H$ up to a logarithmic term, which is an extension of [11]; while the convergence order for the Ait-Sahalia type model is $(2 H-1)\left(\frac{1}{\rho-1} \wedge 1\right)$ up to a logarithmic term.

This paper is structured as follows. In Section 2, we shall recall some basic facts on fractional Brownian motion. Section 3 is devoted to general discussions on (1.1), including existence and uniqueness of solutions to the equation; (negative-power) moments and modular of continuity estimates. In Section 4, we shall present our results on the numerical approximations of (1.1) and their applications on concrete examples.

\section{Preliminaries}

We shall recall some basic facts about fractional Brownian motion. For more details, we refer readers to $[6,23,26]$.

Let $B^{H}=\left\{B_{t}^{H}, t \in[0, T]\right\}$ be a fractional Brownian motion with Hurst parameter $H \in(1 / 2,1)$ defined on the probability space $(\Omega, \mathscr{F}, \mathbb{P})$, i.e. $B^{H}$ is a Gaussian process which is centered with the covariance function

$$
\mathbb{E}\left(B_{t}^{H} B_{s}^{H}\right)=R_{H}(t, s)=\frac{1}{2}\left(t^{2 H}+s^{2 H}-|t-s|^{2 H}\right) .
$$

For each $t \in[0, T]$, let $\mathcal{F}_{t}$ be the $\sigma$-algebra generated by the random variables $\left\{B_{s}^{H}: s \in[0, t]\right\}$ and the sets of probability zero. Furthermore, one can show that $\mathbb{E}\left|B_{t}^{H}-B_{s}^{H}\right|^{p}=C(p)|t-s|^{p H}$ for all $p \geq 1$. As a consequence of the Kolmogorov continuity criterion, $B^{H}$ has $(H-\epsilon)$-order Hölder continuous paths for all $\epsilon>0$. Indeed, the studies on the sample path property of fractional Brownian motion, see for instance [26], show that

$$
\left|B_{t}^{H}-B_{s}^{H}\right| \leq A|t-s|^{H} \sqrt{\log \left(1+(t-s)^{-1}\right)}
$$

where $A$ is a random variable depending on $H$ only and there is some $c>0$ such that $\mathbb{E} e^{c A^{2}}<\infty$. 
Denote by $\mathscr{E}$ the set of step functions on $[0, T]$. Let $\mathcal{H}$ be the Hilbert space defined as the closure of $\mathscr{E}$ with respect to the scalar product

$$
\left\langle I_{[0, t]}, I_{[0, s]}\right\rangle_{\mathcal{H}}:=\alpha_{H} \int_{0}^{T} \int_{0}^{T} \mathbb{1}_{[0, t]}(u) \mathbb{1}_{[0, s]}(v)|u-v|^{2 H-2} \mathrm{~d} u \mathrm{~d} v=R_{H}(t, s),
$$

where $\alpha_{H}=H(2 H-1)$. By the bounded linear transformation theorem, the mapping $I_{[0, t]} \mapsto B_{t}^{H}$ can be extended to an isometry between $\mathcal{H}$ and the Gaussian space associated with $B^{H}$. Denote this isometry by $\phi \mapsto B^{H}(\phi)$.

On the other hand, the covariance kernel $R_{H}(t, s)$ can be written as

$$
R_{H}(t, s)=\int_{0}^{t \wedge s} K_{H}(t, r) K_{H}(s, r) \mathrm{d} r
$$

where $K_{H}$ is a square integrable kernel given by

$$
K_{H}(t, s)=\frac{s^{1 / 2-H}}{\Gamma(H-1 / 2)} \int_{s}^{t} r^{H-1 / 2}(r-s)^{H-3 / 2} \mathrm{~d} r \mathbb{1}_{[0, t]}(s)
$$

in which $\Gamma(\cdot)$ is the Gamma function. Using this kernel, we can define a map from $L^{2}([0, T])$ to the reproducing kernel space $\mathscr{H}$ defined as follows: let

$$
\mathscr{H}=\overline{\operatorname{span}\left\{R_{H}(t, \cdot) \mid t \in[0, T]\right\}}\langle\cdot, \cdot\rangle_{R},
$$

where $\left\langle R_{H}(t, \cdot), R_{H}(s, \cdot)\right\rangle_{R}=R_{H}(t, s), s, t \in[0, T]$, and for any $\phi \in L^{2}([0, T])$, define

$$
\left(K_{H} \phi\right)(t)=\int_{0}^{t} K_{H}(t, s) \phi(s) \mathrm{d} s, t \in[0, T] .
$$

It has been proved in $[4,10]$ that $K_{H}$ is an isomorphism from $L^{2}([0, T])$ to $\mathscr{H}$.

Now, define the linear operator $K_{H}^{*}: \mathscr{E} \rightarrow L^{2}([0, T])$ by

$$
\left(K_{H}^{*} \phi\right)(s)=K_{H}(T, s) \phi(s)+\int_{s}^{T}(\phi(r)-\phi(s)) \frac{\partial K_{H}}{\partial r}(r, s) \mathrm{d} r .
$$

By integration by parts, one can see that

$$
\left(K_{H}^{*} \phi\right)(s)=\int_{s}^{T} \phi(r) \frac{\partial K_{H}}{\partial r}(r, s) \mathrm{d} r .
$$

It is clear that $\left(K_{H}^{*} \mathbb{1}_{[0, t]}\right)(s)=K_{H}(t, s) \mathbb{1}_{[0, t]}(s) . K_{H}^{*}$ is the dual operator of $K_{H}$ in the following sense: for any $\psi \in \mathscr{E}$ and $h \in L^{2}([0, T])$,

$$
\int_{0}^{T}\left(K_{H}^{*} \phi\right)(r) h(t) \mathrm{d} r=\int_{0}^{T} \phi(r)\left(K_{H} h\right)(\mathrm{d} r)
$$

Due to [3], for all $\phi, \psi \in \mathscr{E}$, it holds that $\left\langle K_{H}^{*} \phi, K_{H}^{*} \psi\right\rangle_{L^{2}([0, T])}=\langle\phi, \psi\rangle_{\mathcal{H}}$ and then $K_{H}^{*}$ can be extended to an isometry between $\mathcal{H}$ and $L^{2}([0, T])$. Hence, according 
to [3] again, the process $\left\{W_{t}=B^{H}\left(\left(K_{H}^{*}\right)^{-1} \mathrm{I}_{[0, t]}\right), t \in[0, T]\right\}$ is a Wiener process, and $B^{H}$ has the following integral representation

$$
B_{t}^{H}=\int_{0}^{T}\left(K_{H}^{*} \mathbb{1}_{[0, t]}\right)(s) \mathrm{d} W_{s}=\int_{0}^{t} K_{H}(t, s) \mathrm{d} W_{s} .
$$

With linear operators $K_{H}$ and $K_{H}^{*}$ in hand, there exists an isometry from $\mathcal{H}$ to $\mathscr{H}$ defined by the operator $K_{H} K_{H}^{*}$. Then $\mathscr{H}$ can be charactered by $\mathcal{H}$ with the isometry $K_{H} K_{H}^{*}$. It follows from the integral representation of fBM that $\mathscr{H}$ is the fractional version of the Cameron-Martin space. This was shown rigorously in [10]. The Malliavin derivative of the functional of fBM is defined as an $\mathcal{H}$-valued random variable. For more details on the Malliavin calculus for fBM, one can consult [23].

In this paper, the stochastic integral of fractional Brownian motion is defined by the techniques of fractional calculus developed by Zähle in [27]. We cite the following results on the Riemann-Stieltjes integral and chain rule as a proposition for future use.

Proposition 2.1. Let $a, b \in \mathbb{R}$ with $a<b$, and let $F \in C^{1}(\mathbb{R})$.

(1) Suppose $f \in C^{\lambda}(a, b)$ and $g \in C^{\mu}(a, b)$, where $C^{\lambda}(a, b)$ and $C^{\mu}(a, b)$ are Hölder continuous functions with order $\lambda$ and $\mu$ respectively. If $\lambda+\mu>1$, then the Riemann-Stieltjes integral $\int_{a}^{b} f \mathrm{~d} g$ exists.

(2) Suppose $f \in C^{\lambda}(a, b)$ such that $F^{\prime} \circ f \in C^{\mu}(a, b)$ with $\lambda+\mu>1$. Then

$$
F(f(t))-F(f(s))=\int_{s}^{t} F^{\prime} \circ f(r) \mathrm{d} f(r), s, t \in(a, b) .
$$

Finally, we shall recall a result on the relationship of stochastic integral and the Skorohod integral w.r.t. fractional Brownian motion. Let

$$
|\mathcal{H}|=\left\{\psi \in \mathcal{H}\left|\|\psi\|_{|\mathcal{H}|}^{2}=\alpha_{H} \int_{0}^{T} \int_{0}^{T}\right| \psi(s) \| \psi(t)|| t-\left.s\right|^{2 H-2} \mathrm{~d} s \mathrm{~d} t<\infty\right\}
$$

and $|\mathcal{H}| \otimes|\mathcal{H}|$ be the set of all measurable function such that

$$
\|\psi\|_{|\mathcal{H}| \otimes|\mathcal{H}|}^{2}:=\alpha_{H}^{2} \int_{[0, T]^{4}}|\psi(u, s)||\psi(v, t)||u-v|^{2 H-2}|t-s|^{2 H-2} \mathrm{~d} u \mathrm{~d} v \mathrm{~d} t \mathrm{~d} s<\infty .
$$

For $p>1$, we denote by $\mathbb{D}_{|\mathcal{H}|}^{1, p}$ all the random variables $u$ such that $u \in|\mathcal{H}|$ a.s., its Malliavin derivative $D u \in|\mathcal{H}| \otimes|\mathcal{H}|$ a.s., and

$$
\mathbb{E}\|u\|_{|\mathcal{H}|}^{p}+\mathbb{E}\|D u\|_{|\mathcal{H}| \otimes|\mathcal{H}|}^{p}<\infty
$$

Then we have the following proposition on the link between stochastic integral w.r.t. fBM and the Skorohod integral, see [23, Proposition 5.2.3 and Page 293] and $[2]$. 
Proposition 2.2. Let $H>\frac{1}{2}$, and let $\left\{u_{t}\right\}_{t \in[0, T]}$ be a stochastic process in $\mathbb{D}_{|\mathcal{H}|}^{1,2}$ such that a.s.

$$
\int_{0}^{T} \int_{0}^{T}\left|D_{s} u_{t}\right||t-s|^{2 H-2} \mathrm{~d} t \mathrm{~d} s<\infty
$$

Then

$$
\int_{0}^{T} u_{t} \mathrm{~d} B_{t}^{H}=\delta(u)+\alpha_{H} \int_{0}^{T} \int_{0}^{T} D_{s} u_{t}|t-s|^{2 H-2} \mathrm{~d} t \mathrm{~d} s .
$$

For $p>\frac{1}{H}$,

$$
\mathbb{E}\left(\sup _{t \in[0, T]}\left|\delta\left(u \mathbb{1}_{[0, t]}\right)\right|^{p}\right) \leq C\left(\mathbb{E} \int_{0}^{T}\left|u_{s}\right|^{p} \mathrm{~d} s+\mathbb{E} \int_{0}^{T}\left(\int_{0}^{T}\left|D_{r} u_{s}\right|^{\frac{1}{H}} \mathrm{~d} r\right)^{p H} \mathrm{~d} s\right) .
$$

Remark 2.1. The Proposition 5.2.3 in [23] concerns Stratonovich integral w.r.t. the fBM. For a process $u_{t}$ satisfying the assumption of Proposition 2.2, the same as that of Proposition 5.2.3 in [23], the Stratonovich integral of $u$ w.r.t. the fBM coincides with the forward integrals, see [23, Remark 2 in Page 292]. By the definition of the forward integrals w.r.t. fBM and the link between fractional and stochastic calculus, see [6, Subsection 5.2], the forward integral w.r.t. $f B M$ with $H \in\left(\frac{1}{2}, 1\right)$ is an extension of fractional integral introduced in [27]. In particular, if $u_{t}$ is a $\lambda$-Hölder continuous paths with $\lambda+H>1$, then the integration $\int_{0}^{T} u_{t} \mathrm{~d} B_{t}^{H}$ coincides with the pathwise Riemann-Stieltjes integral.

The second claim of this proposition is the maximal inequality for the divergence integral, see (i) in Page 293 in [23] or [2, Theorem 4]. In the following discussion, we shall make use of the notation

$$
\int_{0}^{t} u_{s} \delta B_{s}^{H}=\delta\left(u \mathbb{1}_{[0, t]}\right)
$$

\section{A study of SDEs driven by fractional Brow- nian motion}

In this section, we shall consider (1.1) following [18]. Fix $T>0$. We prove the existence and uniqueness of this equation on $[0, T]$ under the following assumptions.

(A1) The drift term $b:[0, T] \times(0, \infty) \rightarrow \mathbb{R}$ is continuous and has continuous derivative w.r.t. the second variable. There exists $K \geq 0$ such that

$$
b_{x}^{\prime}(t, x) \leq K, x \in(0, \infty), t \in[0, T],
$$

where $b_{x}^{\prime}$ is the partial derivative of $b$ w.r.t. the second variable.

(A2) There exist $x_{1}>0, \alpha>\frac{1}{H}-1$ and $h_{1}>0$ such that

$$
b(t, x) \geq h_{1} x^{-\alpha}, t \in[0, T], x \leq x_{1} .
$$


(A3) There are $x_{2}>0$ and $h_{2}>0$ such that

$$
b(t, x) \leq h_{2}(x+1), x \geq x_{2}, t \geq 0 .
$$

Remark 3.1. The Assumption (A2) is similar to (ii) in [18]. The assumptions (A1) and (A3) are weaker than (i) and (iii) in [18]. For example, let $b(t, x)=$ $a_{1} x^{-\gamma}-a_{2} x$ with $\gamma \in(1,+\infty), a_{1}>0$ and $a_{2}<0$. It is clear that $b$ satisfies (A1) and (A3), while breaks (i) and (iii) in [18].

Under (A1)-(A3), we prove in Theorem 3.1 below that (1.1) has a positive solution on $(0, T]$ a.s. [21] studies positiveness of solutions to fractional C-I-R models with $H \in(0,1)$ under the framework of pathwise Stratonovich integral. Our result covers [21, Theorem 2], where the authors deal with fractional C-I-R model with $H>\frac{1}{2}$. However, we do not discuss positiveness of solutions to (1.1) with $H<\frac{1}{2}$.

The existence and uniqueness of solutions to (1.1) follows from the existence and uniqueness of the equation below:

$$
\mathrm{d} X_{t}=b\left(t, X_{t}\right) \mathrm{d} t+\mathrm{d} w_{t}, X_{0} \geq 0,
$$

where $w \in C^{\beta}([0, T], \mathbb{R})$ for all $T>0$ with $\beta \in\left(\frac{1}{2}, H\right)$ such that $\alpha>\frac{1}{\beta}-1$. We say $f$ is a $\beta$-Hölder continuous function on $[s, t]$ if

$$
\|f\|_{s, t, \beta}:=\sup _{s \leq s^{\prime}<t^{\prime} \leq t} \frac{\left|f\left(s^{\prime}\right)-f\left(t^{\prime}\right)\right|}{\left(t^{\prime}-s^{\prime}\right)^{\beta}}<\infty .
$$

Sometimes, we use $\|\cdot\|_{\beta}$ for simplicity's sake. For a continuous function $f$ on $[s, t]$, we define

$$
\|f\|_{s, t, \infty}=\sup _{s \leq r \leq t}\left|f_{r}\right| .
$$

Our existence and uniqueness theorem for (3.1) reads as follows.

Theorem 3.1. Assume that (A1)-(A3) hold.

(1) For all $X_{0}>0$, it holds that the equation (3.1) has a unique solution $X_{t}$ and $X \in C^{\beta}([0, T],(0, \infty))$

(2) For $X_{0}=0$, if there exists $t_{0}>0$ such that $b(t, \cdot)$ is non-increasing on $\left(0, x_{1}\right)$ for all $0 \leq t \leq t_{0}$, then (3.1) has a unique solution $X_{t}$ and $X_{t} \in$ $(0, \infty)$ for all $t \in[0, T]$.

Proof. We first prove the uniqueness. Let $X_{t}^{[1]}$ and $X_{t}^{[2]}$ be two solutions of equation (3.1) with the same initial values, then

$$
X_{t}^{[1]}-X_{t}^{[2]}=X_{s}^{[1]}-X_{s}^{[2]}+\int_{s}^{t}\left(b\left(r, X_{r}^{[1]}\right)-b\left(r, X_{r}^{[2]}\right)\right) \mathrm{d} r, s \leq t \leq T .
$$

Combining this with (A1), we have

$$
\mathrm{d}\left(X_{t}^{[1]}-X_{t}^{[2]}\right)^{2}=2\left(b\left(t, X_{t}^{[1]}\right)-b\left(t, X_{t}^{[2]}\right)\right)\left(X_{t}^{[1]}-X_{t}^{[2]}\right) \mathrm{d} t
$$




$$
\leq 2 K\left(X_{t}^{[1]}-X_{t}^{[2]}\right)^{2} \mathrm{~d} t
$$

which yields

$$
\left(X_{t}^{[1]}-X_{t}^{[2]}\right)^{2} \leq \exp (2 K t)\left(X_{0}^{[1]}-X_{0}^{[2]}\right)^{2}
$$

Thus, it $X_{t}^{[1]}-X_{t}^{[2]}=0$ for all $t \in[0, T]$.

We assume that $X_{0}>0$. Since $b:[0, T] \times(0, \infty) \rightarrow \mathbb{R}$ is continuous and has continuous derivative w.r.t. the second variable, it is clear that (3.1) has a continuous local solution. Next, we shall prove that $X_{t} \in(0, \infty)$ for all $t \in[0, T]$. Let

$$
\tau_{0}=\inf \left\{t \in[0, T] \mid X_{t}=0\right\}, \quad \tau_{n}=\inf \left\{t \in[0, T] \mid X_{t} \geq n\right\}, n \in \mathbb{N},
$$

with the convention that $\inf \emptyset:=T+$, where $T+$ is an artificial added element larger than $T$, but smaller than any $a>T$. We shall prove $\tau_{0}=T+$ and $\lim _{n \rightarrow \infty} \tau_{n}=T+$.

If $\tau_{0} \leq T$, then there is $\hat{\tau}_{0} \in\left(0, \tau_{0}\right)$ such that $X_{t} \leq x_{1}$ for all $t \in\left(\hat{\tau}_{0}, \tau_{0}\right]$. Since $b(t, x)>0$ for $x \in\left(0, x_{1}\right), t \geq 0$ and

$$
0=X_{\tau_{0}}=X_{t}+\int_{t}^{\tau_{0}} b\left(s, X_{s}\right) \mathrm{d} s+w_{\tau_{0}}-w_{t}
$$

it follows that

$$
X_{t} \leq\left|w_{\tau_{0}}-w_{t}\right| \leq\|w\|_{\beta}\left(\tau_{0}-t\right)^{\beta}, t \in\left(\hat{\tau}_{0}, \tau_{0}\right) .
$$

On the other hand, by (A2), (3.2) and (3.3), we have

$$
\begin{aligned}
\|w\|_{\beta}\left(\tau_{0}-t\right)^{\beta} & \geq\left|w_{\tau_{0}}-w_{t}\right| \geq \int_{t}^{\tau_{0}} b\left(s, X_{s}\right) \mathrm{d} s \\
& \geq h_{1} \int_{t}^{\tau_{0}} X_{s}^{-\alpha} \mathrm{d} s \geq \frac{h_{1}}{\|w\|_{\beta}^{\alpha}} \int_{t}^{\tau_{0}} \frac{1}{\left(\tau_{0}-s\right)^{\alpha \beta}} \mathrm{d} s, t>\hat{\tau}_{0} .
\end{aligned}
$$

If $\alpha \beta \geq 1$, then $\int_{t}^{\tau_{0}} \frac{1}{\left(\tau_{0}-s\right)^{\alpha \beta}} \mathrm{d} s=\infty$ which leads to a contradiction. If $\alpha \beta<1$, then

$$
\|w\|_{\beta}\left(\tau_{0}-t\right)^{\beta} \geq \frac{\left(\tau_{0}-t\right)^{1-\alpha \beta} h_{1}}{(1-\alpha \beta)\|w\|_{\beta}^{\alpha}}, t>\hat{\tau}_{0} .
$$

This, together with $\alpha>\frac{1}{\beta}-1$, implies that

$$
0=\lim _{t \rightarrow \tau_{0}^{-}}\left(\tau_{0}-t\right)^{\alpha \beta+\beta-1} \geq \frac{h_{1}}{\|w\|_{\beta}^{\alpha+1}}>0,
$$

which is a contradiction. Hence $\tau_{0}=T+$. 
If $\tau_{\infty}:=\lim _{n \rightarrow \infty} \tau_{n} \leq T$, then either there exists $\hat{\tau}_{1}$ such that $X_{\hat{\tau}_{1}}=x_{2}+X_{0}$ and $X_{t} \geq x_{2}+X_{0}$ for all $t \in\left(\hat{\tau}_{1}, \tau_{\infty}\right)$, or for all $n \in \mathbb{N}$ with $n>x_{2}+X_{0}$ and $\epsilon>0$ there exits an interval $\left(\hat{\tau}_{1}, \hat{\tau}_{2}\right) \subset\left(\tau_{\infty}-\epsilon, \tau_{\infty}\right)$ such that $X_{\hat{\tau}_{1}}=x_{2}+X_{0}$ and

$$
x_{2}+X_{0} \leq \inf _{t \in\left(\hat{\tau}_{1}, \hat{\tau}_{2}\right)} X_{t} \leq n \leq \sup _{t \in\left(\hat{\tau}_{1}, \hat{\tau}_{2}\right)} X_{t}
$$

In both cases,

$$
\begin{aligned}
X_{t} & =X_{\hat{\tau}_{1}}+\int_{\hat{\tau}_{1}}^{t} b\left(s, X_{s}\right) \mathrm{d} s+w_{t}-w_{\hat{\tau}_{1}} \\
& \leq x_{2}+X_{0}+\int_{\hat{\tau}_{1}}^{t} h_{2}\left(X_{s}+1\right) \mathrm{d} s+w_{t}-w_{\hat{\tau}_{1}} \\
& \leq x_{2}+X_{0}+\|w\|_{\beta} \tau_{\infty}^{\beta}+h_{2} \tau_{\infty}+h_{2} \int_{\hat{\tau}_{1}}^{t} X_{s} \mathrm{~d} s .
\end{aligned}
$$

where we use (A3) in the second inequality. It follows from Grönwall's inequality that for all $t \in\left(\hat{\tau}_{1}, \hat{\tau}_{2}\right)$ or $t \in\left(\hat{\tau}_{1}, \tau_{\infty}\right)$

$$
\begin{aligned}
X_{t} & \leq\left(x_{2}+X_{0}+\|w\|_{\beta} \tau_{\infty}^{\beta}+h_{2} \tau_{\infty}\right) \exp \left\{\left(t-\hat{\tau}_{1}\right) h_{2}\right\} \\
& \leq\left(x_{2}+X_{0}+\|w\|_{\beta} T^{\beta}+h_{2} T\right) e^{T h_{2}} .
\end{aligned}
$$

Taking supremum of the left hand side in the above inequality: for all $t \in$ $\left(\hat{\tau}_{1}, \tau_{\infty}\right)$ in the first case or for all $t \in\left(\hat{\tau}_{1}, \hat{\tau}_{2}\right), \epsilon \in(0,1)$, and $n \geq 1$ for the second case, the left hand side is infinite but the right hand side is a finite constant. This is a contradiction. Hence, $\tau_{\infty}=T+$.

Finally, we deal with the case $X_{0}=0$. For $n \in \mathbb{N}$, let $X_{t}^{[n]}$ be the solution of (3.1) with $X_{0}^{[n]}=1 / n$. For $n, m \in \mathbb{N}, n<m$, let $\tau=\inf \left\{t \in[0, T] \mid X_{t}^{[n]}=\right.$ $\left.X_{t}^{[m]}\right\}$. By the uniqueness, $X_{t}^{[n]}=X_{t}^{[m]}$ for all $t \geq \tau$, or $\tau=T+$. It is clear that $X_{t}^{[n]}>X_{t}^{[m]}$ if $t<\tau$. Thus, the sequence $\left\{X_{t}^{[n]}\right\}_{n \in \mathbb{N}}$ is non-increasing and nonnegative. Let $n_{0} \in \mathbb{N}$ be such that $\frac{1}{n_{0}}<x_{1}$, and let $\tau^{n_{0}}=\inf \{t \in$ $\left.[0, T] \mid X_{t}^{\left[n_{0}\right]} \geq x_{1}\right\}$. Set $X_{t}=\lim _{n \rightarrow \infty} X_{t}^{[n]}$. Then

$$
X_{t \wedge \tau^{n_{0}}} \leq X_{t \wedge \tau^{n_{0}}}^{[n]} \leq X_{t \wedge \tau^{n_{0}}}^{\left[n_{0}\right]} \leq x_{1}, n \geq n_{0}
$$

Set $b(t, 0)=+\infty$ for $t \in\left[0, t_{0}\right]$. Then

$$
b\left(t, X_{t}\right)=\lim _{n \rightarrow+\infty} b\left(t, X_{t}^{[n]}\right), t \in\left(0, t_{0} \wedge \tau^{n_{0}}\right]
$$

Since for any $t \in\left[0, t_{0}\right], b(t, x)$ is non-increasing for $x \in\left(0, x_{1}\right)$, the following equality follows from the monotone convergence theorem

$$
\lim _{n \rightarrow \infty} \int_{0}^{t \wedge t_{0} \wedge \tau^{n_{0}}} b\left(s, X_{s}^{[n]}\right) \mathrm{d} s=\int_{0}^{t \wedge t_{0} \wedge \tau^{n_{0}}} b\left(s, X_{s}\right) \mathrm{d} s .
$$


Taking into account that $X_{t}^{[n]}$ satisfies (3.1), we have

$$
X_{t \wedge t_{0} \wedge \tau^{n_{0}}}=\int_{0}^{t \wedge t_{0} \wedge \tau^{n_{0}}} b\left(s, X_{s}\right) \mathrm{d} s+w_{t \wedge t_{0} \wedge \tau^{n_{0}}}-w_{0} .
$$

Moreover, this inequality yields that

$$
\int_{0}^{t \wedge t_{0} \wedge \tau^{n_{0}}} b\left(s, X_{s}\right) \mathrm{d} s<\infty .
$$

Thus, $b\left(s, X_{s}\right)<\infty$ a.e. $s \in\left[0, t \wedge t_{0} \wedge \tau^{n_{0}}\right]$. By (A2), $X_{s}>0$ a.e. $s \in$ $\left[0, t \wedge t_{0} \wedge \tau^{n_{0}}\right]$. Starting from any $X_{s}>0$ with $s \in\left(0, t \wedge t_{0} \wedge \tau^{n_{0}}\right)$, there exists unique solution to (3.1) which is positive. Thus, $X_{s}>0$ for all $s \in\left(0, t \wedge t_{0} \wedge \tau^{n_{0}}\right]$. According to the proof above, $\left\{X_{t}\right\}_{t \in\left[0, t \wedge t_{0} \wedge \tau^{n_{0}}\right]}$ can be extended to a solution for all $t>0$ and $X_{t}>0$ for all $t \in(0, T]$.

Remark 3.2. It is clear that $X \in C^{\beta}([s, T])$ for all $0 \leq s<T$ only if $X_{0}>0$ or $s>0$. In [18, Remark 2.2], the authors stated that the solution of (2.1) in [18] is in $C^{\beta}([0, T])$ even if the initial value is 0 . However, we should point out that the positiveness of the initial value is necessary. In fact, the solution $X_{t}$ with $X_{0}=0$ is not $\beta$-Hölder continuous on the interval which contains 0 . Otherwise, there is $C>0$ and $t_{1}>0$ such that $X_{t} \leq C t^{\beta}$ for $t \in\left[0, t_{1}\right]$. Letting $\tau_{x_{1}}=\inf \left\{t \geq 0 \mid X_{t} \geq x_{1}\right\}$, just as (3.4), it follows from (3.1) and (A2) that for $t \leq \tau_{x_{1}}$ we have

$$
X_{t}=X_{0}+\int_{0}^{t} b\left(s, X_{s}\right) \mathrm{d} s+w_{t}-w_{0} \geq h_{1} \int_{0}^{t} \frac{1}{C^{\alpha} s^{\beta \alpha}} \mathrm{d} s-\|w\|_{\beta} t^{\beta} .
$$

As in proof of $\tau_{0}=T+,(3.6)$ leads to a contradiction if $\alpha \beta \geq 1$. For $\alpha \beta<1$, it is similar to (3.5) that (3.6) and $X_{t} \leq C t^{\beta}$ lead to the following contradiction

$$
C+\|w\|_{\beta} \geq \varlimsup_{t \rightarrow 0^{+}} \frac{X_{t}+\|w\|_{\beta} t^{\beta}}{t^{\beta}} \geq \frac{h_{1}}{C} \varlimsup_{t \rightarrow 0^{+}} t^{1-\alpha \beta-\beta}=\infty .
$$

According to this theorem, the stochastic equation (1.1) has a unique pathwise solution. Next, we shall study the Malliavin differentiablity of $X_{t}$.

Lemma 3.2. Assume (A1), (A2) and (A3) hold. Let $X_{t}$ be the solution of (1.1). Then for all $t>0, X_{t} \in \mathbb{D}_{|\mathcal{H}|}^{1,2}$ with

$$
D_{s} X_{t}=\sigma \exp \left\{\int_{s}^{t} b_{x}^{\prime}\left(r, X_{r}\right) \mathrm{d} r\right\} \mathbb{1}_{[0, t]}(s),
$$

and the law of $X_{t}$ has density w.r.t. the Lebesgue measure on $\mathbb{R}$.

The proof just follows the line of [18, Theorem 3.3.], and the outline of the proof is presented here for the convenience of readers. 
Proof. Let $\epsilon \in(0,1), h \in \mathcal{H}$ with $h_{0}=0$ and

$$
X_{t}^{\epsilon}=X_{0}+\int_{0}^{t} b\left(r, X_{r}^{\epsilon}\right) \mathrm{d} r+\sigma B_{t}^{H}+\sigma \epsilon K_{H} K_{H}^{*} h(t) .
$$

Then by (A1),

$$
\begin{aligned}
X_{t}^{\epsilon}-X_{t} & =\int_{0}^{t}\left(b\left(r, X_{r}^{\epsilon}\right)-b\left(r, X_{r}\right)\right) \mathrm{d} r+\sigma \epsilon K_{H} K_{H}^{*} h(t) \\
& =\int_{0}^{t} b_{x}^{\prime}\left(r, X_{r}^{\xi}\right)\left(X_{r}^{\epsilon}-X_{r}\right) \mathrm{d} r+\sigma \epsilon K_{H} K_{H}^{*} h(t), t>0,
\end{aligned}
$$

where $X_{r}^{\xi}=X_{r}+\xi_{s}^{\epsilon}\left(X_{r}^{\epsilon}-X_{r}\right)$ and $\xi_{s}^{\epsilon} \in(0,1)$ depends on $s$ and $\epsilon$. This equality, along with (2.1) (see also [6, Lemma 2.1.9]), implies

$$
\begin{aligned}
X_{t}^{\epsilon}-X_{t} & =\sigma \epsilon \int_{0}^{t} \exp \left\{\int_{s}^{t} b_{x}^{\prime}\left(r, X_{r}^{\xi}\right) \mathrm{d} r\right\}\left(K_{H} K_{H}^{*} h\right)(\mathrm{d} s) \\
& =\sigma \epsilon \int_{0}^{T} K_{H}^{*}\left(\exp \left\{\int^{t} b_{x}^{\prime}\left(r, X_{r}^{\xi}\right) \mathrm{d} r\right\} \mathbb{1}_{[0, t]}(\cdot)\right)(s) K_{H}^{*} h(s) \mathrm{d} s .
\end{aligned}
$$

Since the continuity of $b_{x}^{\prime}(t, \cdot),(\mathbf{A} 1)$ and $K_{H}^{*} h \in L^{2}([0, T])$, it follows from the dominated convergence theorem that the limit

$$
\begin{aligned}
\lim _{\epsilon \rightarrow 0^{+}} \frac{X_{t}^{\epsilon}-X_{t}}{\epsilon} & =\sigma \int_{0}^{T} K_{H}^{*}\left(\exp \left\{\int^{t} b_{x}^{\prime}\left(r, X_{r}\right) \mathrm{d} r\right\} \mathbb{1}_{[0, t]}(\cdot)\right)(s) K_{H}^{*} h(s) \mathrm{d} s \\
& =\sigma\left\langle\exp \left\{\int^{t} b_{x}^{\prime}\left(r, X_{r}\right) \mathrm{d} r\right\} \mathbb{1}_{[0, t]}(\cdot), h\right\rangle_{\mathcal{H}}
\end{aligned}
$$

holds almost sure and in $L^{2}(\Omega)$. Consequently,

$$
D \cdot X_{t}=\sigma \exp \left\{\int^{t} b_{x}^{\prime}\left(r, X_{r}\right) \mathrm{d} r\right\} \mathbb{1}_{[0, t]}(\cdot) .
$$

It is clear that $\left\|D X_{t}\right\|_{\mathcal{H}}>0$, and $\mathbb{E}\left\|D X_{t}\right\|_{\mathcal{H}}^{2}<\infty$ follows from (A1). Then the existence of density w.r.t. the Lebesgue measure follows from the classical result of Malliavin calculus, see e.g. [23, Theorem 2.1.2 or Theorem 2.1.3].

Next, we shall study the moment estimates of solutions to (1.1). To this end, we introduce the following assumption.

(A2') The condition (A2) holds with $\alpha \geq 1$. There exist $\theta>0$ and $h_{3}>0$ such that

$$
b(t, x) \leq h_{3}\left(1+x+x^{-\theta}\right), t \in[0, T], x>0 .
$$

It should be noted that $\theta \geq \alpha$ by (A2) and (3.7), and (A2') implies (A3). This assumption is used for positive moment estimate. To give the negative moment estimate, we introduce the following 
(A3') there exists a $q>0$ and $h_{4}>0$ such that

$$
(b(t, x))^{-} \leq h_{4}\left(1+x^{q}\right), s \in[0, T], x>0
$$

where $(b(t, x))^{-}$denotes the negative part of $b(t, x)$.

We first consider the negative moments for the solution to (1.1).

Lemma 3.3. Assume (A1), (A2') and (A3'). Let $X_{t}$ be a solution to (1.1) with $X_{0}>0$.

(1) Suppose $\alpha=1$. Then for $p \geq 1$ and

$$
h_{1} \geq((p+1) \vee q) H T^{2 H-1} e^{K T},
$$

one has

$$
\sup _{s \in[0, T]} \mathbb{E} X_{s}^{-p}<\infty .
$$

If (3.9) holds with $p$ replaced by $2(p+2)$, then

$$
\mathbb{E} \sup _{s \in[0, T]} X_{s}^{-p}<\infty
$$

(2) Suppose $\alpha>1$. Then for all $p>0$,

$$
\mathbb{E} \sup _{s \in[0, T]} X_{s}^{-p}<\infty
$$

Proof. We divide the proof into two steps

Step (i). We first prove (3.10). In fact, due to the Hölder inequality, we only need to prove the claim for large $p$. Thus we assume that $p+1 \geq q$. Since $X_{t}$ is $\beta$-Hölder continuous for $\beta<H$, applying Proposition 2.1, Proposition 2.2 and Lemma 3.2, we obtain that

$$
\begin{aligned}
\left(X_{t}+\epsilon\right)^{-p}= & \left(X_{0}+\epsilon\right)^{-p}-p \int_{0}^{t} \frac{b\left(s, X_{s}\right)}{\left(\epsilon+X_{s}\right)^{p+1}} \mathrm{~d} s-\sigma p \int_{0}^{t}\left(\epsilon+X_{s}\right)^{-(p+1)} \mathrm{d} B_{s}^{H} \\
\leq & \left(X_{0}+\epsilon\right)^{-p}-p \int_{0}^{t} \frac{b\left(s, X_{s}\right)}{\left(\epsilon+X_{s}\right)^{p+1}} \mathrm{~d} s-\sigma p \int_{0}^{t}\left(\epsilon+X_{s}\right)^{-(p+1)} \delta B_{s}^{H} \\
& \quad+\sigma p(p+1) \alpha_{H} \int_{0}^{t} \int_{0}^{s} \frac{D_{r} X_{s}|s-r|^{2 H-2}}{\left(\epsilon+X_{s}\right)^{p+2}} \mathrm{~d} r \mathrm{~d} s \\
\leq & \left(X_{0}+\epsilon\right)^{-p}-p \int_{0}^{t} \frac{b\left(s, X_{s}\right) X_{s}-\sigma^{2}(p+1) H s^{2 H-1} e^{K s}}{\left(\epsilon+X_{s}\right)^{p+2}} \mathrm{~d} s \\
& \quad-\sigma p \int_{0}^{t}\left(\epsilon+X_{s}\right)^{-(p+1)} \delta B_{s}^{H} .
\end{aligned}
$$

Let

$$
\tilde{x}_{1}=x_{1} \wedge 1 \wedge\left(\frac{e^{-K T} h_{1}}{\sigma^{2}(p+1) H T^{2 H-1}}\right)^{\frac{1}{\alpha-1}} \mathbb{1}_{[\alpha>1]}+\left(x_{1} \wedge 1\right) \mathbb{1}_{[\alpha=1]} .
$$


Then

$$
\begin{aligned}
-\frac{b(s, x)}{(\epsilon+x)^{p+2}} & \leq-\mathbb{1}_{\left[x \leq \tilde{x}_{1}\right]} \frac{h_{1}}{x^{\alpha}(\epsilon+x)^{p+2}}+\mathbb{1}_{\left[x \geq \tilde{x}_{1}\right]} \frac{h_{4}\left(1+x^{q}\right)}{(\epsilon+x)^{p+2}} \\
& \leq h_{4}\left(\frac{1}{\tilde{x}_{1}^{p+2}}+\frac{1}{\tilde{x}_{1}^{p+2-q}}\right)
\end{aligned}
$$

and

$$
\begin{aligned}
- & \frac{b(s, x) x-\sigma^{2}(p+1) H s^{2 H-1} e^{\int_{0}^{s} K_{u}^{+} \mathrm{d} u}}{(\epsilon+x)^{p+2}} \\
\leq- & \frac{h_{1} x^{-(\alpha-1)}-\sigma^{2}(p+1) H s^{2 H-1} e^{K s}}{(\epsilon+x)^{p+2}} \mathbb{1}_{\left[x \leq \tilde{x}_{1}\right]} \\
& \quad+\frac{h_{4}\left(1+x^{q}\right) x+(p+1) H s^{2 H-1} e^{K s}}{(\epsilon+x)^{p+2}} \mathbb{1}_{\left[x \geq \tilde{x}_{1}\right]} \\
\leq- & \frac{h_{1} \tilde{x}_{1}^{-\alpha+1}-\sigma^{2}(p+1) H s^{2 H-1} e^{K s}}{x^{p+2}} \mathbb{1}_{\left[x \leq \tilde{x}_{1}\right]} \\
& \quad+(p+1) H s^{2 H-1} e^{K s} \tilde{x}_{1}^{-p-2}+h_{4}\left(\tilde{x}_{1}^{-(p+1)}+\tilde{x}_{1}^{-p-1+q}\right) .
\end{aligned}
$$

Since (3.9) and the definition of $\tilde{x}_{1}$, there exists $C>0$ depending on $\tilde{x}_{1}, p, q, \sigma$ such that

$$
\left(X_{t}+\epsilon\right)^{-p} \leq\left(X_{0}+\epsilon\right)^{-p}+C \int_{0}^{t}\left(h_{4}+s^{2 H-1}\right) \mathrm{d} s-\sigma p \int_{0}^{t}\left(\epsilon+X_{s}\right)^{-(p+1)} \delta B_{s}^{H} .
$$

Taking expectation and letting $\epsilon \rightarrow 0$, (3.10) is proved.

Step (ii) We shall complete the rest of the proof. If $\alpha>1$ or (3.9) holds with $p$ replaced by $2(p+2)$, then

$$
\sup _{[0, T]} \mathbb{E} X_{t}^{-2(p+2)}<\infty .
$$

Then $X^{-(p+1)} \in \mathbb{D}_{|\mathcal{H}|}^{1,2}$, (see Page 2 for the definition of $\mathbb{D}_{|\mathcal{H}|}^{1,2}$ ), due to the following inequality

$$
\begin{aligned}
\int_{[0, T]^{2}} & \int_{[0, T]^{2}} \mathbb{E}\left(D_{s} X_{t}\right) X_{t}^{-(p+2)}\left(D_{v} X_{u}\right) X_{u}^{-(p+2)}|u-v|^{2 H-2}|t-s|^{2 H-2} \mathrm{~d} u \mathrm{~d} v \mathrm{~d} s \mathrm{~d} t \\
& \leq \sigma^{2} e^{2 K T} \sup _{[0, T]} \mathbb{E} X_{t}^{-2(p+2)} \int_{[0, T]^{2}} \int_{[0, T]^{2}}|u-v|^{2 H-2}|t-s|^{2 H-2} \mathrm{~d} u \mathrm{~d} v \mathrm{~d} s \mathrm{~d} t \\
& <\infty
\end{aligned}
$$

By Proposition 2.1, Proposition 2.2 and Lemma 3.2 again, there is some $C>0$ depending on $\tilde{x}_{1}, p, q, \sigma$ such that

$$
X_{t}^{-p} \leq X_{0}^{-p}+C \int_{0}^{t}\left(h_{4}+s^{2 H-1}\right) \mathrm{d} s-p \int_{0}^{t} X_{s}^{-(p+1)} \delta B_{s}^{H} .
$$


It follows from the maximal inequality of the Skorohod integral (see e.g. [23, Page 293] or Proposition 2.2) that

$$
\begin{aligned}
& \left(\mathbb{E} \sup _{r \in[0, t]}\left|\int_{0}^{r} X_{s}^{-(p+1)} \delta B_{s}^{H}\right|^{2}\right)^{\frac{1}{2}} \\
& \quad \leq C\left(\int_{0}^{t} \mathbb{E} X_{s}^{-2(p+1)} \mathrm{d} s+\mathbb{E} \int_{0}^{t}\left(\int_{0}^{s}(p+1)^{\frac{1}{H}} X_{s}^{-\frac{p+2}{H}}\left|D_{r} X_{s}\right|^{\frac{1}{H}} \mathrm{~d} r\right)^{2 H} \mathrm{~d} s\right)^{\frac{1}{2}} \\
& \quad \leq C_{p, H}\left(1+t^{H}\right) e^{K t}\left(\int_{0}^{t} \mathbb{E} X_{s}^{-2(p+2)}\left(1+X_{s}^{2}\right) \mathrm{d} s\right)^{\frac{1}{2}} .
\end{aligned}
$$

Then

$$
\begin{aligned}
\mathbb{E} \sup _{s \in[0, T]} X_{s}^{-p} \leq X_{0}^{-p}+C \int_{0}^{T}\left(h_{4}+s^{2 H-1}\right) \mathrm{d} s \\
\quad+C_{p, H}\left(1+T^{H}\right) e^{K T}\left(\int_{0}^{T} \mathbb{E} X_{s}^{-2(p+2)}\left(1+X_{s}^{2}\right) \mathrm{d} s\right)^{\frac{1}{2}},
\end{aligned}
$$

which implies the required conclusion.

If (3.7) holds with $b(t, x)$ replaced by $|b(t, x)|$, then we can obtain moment estimates of $|X|_{0, T, \infty}$ by applying [12, Theorem 3.1] to $X_{t}^{1+\theta}$. However, if (3.8) holds, that is we allow that $|b(t, x)|$ has super-linear growth near infinity, then the following lemma can not be covered by [12]. For $g \in C\left([0, T], \mathbb{R}^{d}\right)$, we denote by $\mathbb{M}_{g, T}(\cdot)$ the modulus of continuity of $g$ on $[0, T]$, i.e.

$$
\mathbb{M}_{g, T}(h)=\sup _{0 \leq s, t \leq T,|s-t| \leq h}\left|g_{t}-g_{s}\right| .
$$

We now give the estimates for the positive moments of the solution to (1.1).

Lemma 3.4. Assume (A1), (A2') and (A3'). Let $\left\{X_{t}\right\}_{t \in[0, T]}$ be a solution of (1.1) with $X_{0}>0$.

(1) If $\alpha>1$, then for any $p>0$, we have

$$
\mathbb{E}\|X\|_{0, T, \infty}^{p}<\infty
$$

and

$$
\left(\mathbb{E M}_{X, T}^{p}(h)+\mathbb{E M}_{X^{-1}, T}^{p}(h)\right)^{\frac{1}{p}} \leq C_{p, T}\left(h+h^{H} \sqrt{\log (1+1 / h)}\right) .
$$

(2) If $\alpha=1$, then for $p>0$, there exists $T>0$ such that (3.11) and (3.12) hold. 
Proof. Suppose $\alpha>1$. We first prove that

$$
\mathbb{E}\left(X_{t}^{p}+\int_{0}^{t} X_{s}^{p} \mathrm{~d} s\right)<\infty, t \geq 0, p>0
$$

By (2) of Proposition 2.1, Lemma 3.2 and Proposition 2.2, for any $n>0$

$$
\begin{aligned}
\frac{n X_{t}^{p}}{n+X_{t}^{p}}- & \frac{n X_{0}^{p}}{n+X_{0}^{p}} \\
= & \int_{0}^{t} \frac{p n^{2} X_{s}^{p-1}}{\left(n+X_{s}^{p}\right)^{2}} b\left(s, X_{s}\right) \mathrm{d} s+\int_{0}^{t} \frac{\sigma p n^{2} X_{s}^{p-1}}{\left(n+X_{s}^{p}\right)^{2}} \mathrm{~d} B_{s}^{H} \\
\leq & \int_{0}^{t} \frac{p n^{2} h_{3} X_{s}^{p-1}\left(1+X_{s}+X_{s}^{-\theta}\right)}{\left(n+X_{s}^{p}\right)^{2}} \mathrm{~d} s+\int_{0}^{t} \frac{\sigma p n^{2} X_{s}^{p-1}}{\left(n+X_{s}^{p}\right)^{2}} \delta B_{s}^{H} \\
& \quad+\int_{0}^{t} \int_{0}^{s} \frac{\alpha_{H} \sigma p n^{2} X_{s}^{p-2}\left(n(p-1)^{+}-(p+1) X_{s}^{p}\right)}{\left(n+X_{s}^{p}\right)^{3}} D_{r} X_{s}|r-s|^{2 H-2} \mathrm{~d} r \mathrm{~d} s \\
\leq & \int_{0}^{t}\left(\frac{2 p n h_{3} X_{s}^{p}}{n+X_{s}^{p}}+p h_{3}\left(1+X_{s}^{-\theta}\right)\right) \mathrm{d} s+\int_{0}^{t} \frac{\sigma p n^{2} X_{s}^{p-1}}{\left(n+X_{s}^{p}\right)^{2}} \delta B_{s}^{H} \\
& \quad+C_{H} e^{K t} \int_{0}^{t} \frac{\sigma^{2} p(p-1)^{+} n X_{s}^{p-2}}{n+X_{s}^{p}} s^{2 H-1} \mathrm{~d} s \\
\leq & C_{t, p, K, H, \sigma} \int_{0}^{t}\left(\frac{n X_{s}^{p}}{n+X_{s}^{p}}\left(h_{3}+s^{2 H-1}\right)\right) \mathrm{d} s+\int_{0}^{t} \frac{\sigma p n^{2} X_{s}^{p-1}}{\left(n+X_{s}^{p}\right)^{2}} \delta B_{s}^{H} \\
\quad & \quad C_{t, p, K, H, \sigma} \int_{0}^{t}\left(h_{3}\left(1+X_{s}^{-\theta}\right)+(p-1)^{+} s^{2 H-1} X_{s}^{-2}\right) \mathrm{d} s,
\end{aligned}
$$

where $C_{t, p, K, H, t}$ is locally bounded in $t$. Then it follows from the Grönwall lemma and Lemma 3.3 that

$$
\mathbb{E} \frac{n X_{t}^{p}}{n+X_{t}^{p}} \leq X_{0}^{p}+e^{C_{t, p, K, H, \sigma}} \int_{0}^{t} \mathbb{E}\left(h_{3}\left(1+X_{s}^{-\theta}\right)+\frac{(p-1)^{+} s^{2 H-1}}{X_{s}^{2}}\right) \mathrm{d} s<\infty,
$$

which implies (3.13) by letting $n \rightarrow \infty$ and using Fatou's lemma.

Next, we shall prove that

$$
\mathbb{E} \sup _{s \in[0, t]} X_{s}^{p}<\infty, t \geq 0, p>0
$$

Indeed, by chain rule, (3.13) and Lemma 3.2, we have $X_{t}^{p-1} \in \mathbb{D}_{|\mathcal{H}|}^{1,2}$ and

$$
\begin{aligned}
X_{t}^{p}=X_{0}^{p} & +\int_{0}^{t} X_{s}^{p-1} b\left(s, X_{s}\right) \mathrm{d} s+\sigma \int_{0}^{t} X_{s}^{p-1} \mathrm{~d} B_{s}^{H} \\
\leq X_{0}^{p} & +\int_{0}^{t} h_{3}\left(X_{s}^{p-1}+X_{s}^{p}+X_{s}^{p-\theta-1}\right) \mathrm{d} s+\sigma \int_{0}^{t} X_{s}^{p-1} \delta B_{s}^{H} \\
& \quad+\sigma(p-1) \alpha_{H} \int_{0}^{t} \int_{0}^{s} X_{s}^{p-s} D_{r} X_{s}|r-s|^{2 H-2} \mathrm{~d} r \mathrm{~d} s
\end{aligned}
$$




$$
\begin{aligned}
\leq X_{0}^{p} & +C \int_{0}^{t} h_{3}\left(1+X_{s}^{p}\right) \mathrm{d} s+|\sigma|\left|\int_{0}^{t} X_{s}^{p-1} \delta B_{s}^{H}\right| \\
& +C_{H, \sigma, p} e^{K t} \int_{0}^{t} X_{s}^{p-2}|s|^{2 H-1} \mathrm{~d} s .
\end{aligned}
$$

The maximal inequality of Skorohod integral yields that the following inequality holds

$$
\begin{aligned}
& \left(\mathbb{E} \sup _{s \in[0, t]}\left|\int_{0}^{s} X_{r}^{p-1} \delta B_{r}^{H}\right|^{2}\right)^{\frac{1}{2}} \\
& \quad \leq C\left(\int_{0}^{t} \mathbb{E} X_{r}^{2(p-1)} \mathrm{d} r+\mathbb{E} \int_{0}^{t}\left(\int_{0}^{r}(p-1)^{\frac{1}{H}} X_{r}^{\frac{p-2}{H}}\left|D_{u} X_{r}\right|^{\frac{1}{H}} \mathrm{~d} u\right)^{2 H} \mathrm{~d} r\right)^{\frac{1}{2}} \\
& \quad \leq C_{H, p}\left(1+t^{H}\right) e^{K t}\left(\int_{0}^{t} \mathbb{E} X_{r}^{2(p-2)}\left(1+X_{s}^{2}\right) \mathrm{d} r\right)^{\frac{1}{2}}
\end{aligned}
$$

Combining (3.15) and (3.16) with (3.13), we get (3.14).

Next, we shall give the estimates of modulus of continuity. By (A2') and (A3'), we have

$$
|b(s, x)| \leq\left(h_{4} \vee h_{3}\right)\left(1+x^{q}+x^{-\theta}\right) \equiv \tilde{h}\left(1+x^{q}+x^{-\theta}\right) .
$$

Then for any $t>s \geq 0$,

$$
\begin{aligned}
\left|X_{t}-X_{s}\right| \leq \int_{s}^{t}\left|b\left(r, X_{r}\right)\right| \mathrm{d} r+\left|\sigma\left(B_{t}^{H}-B_{s}^{H}\right)\right| \\
\leq \int_{s}^{t} \tilde{h}\left(1+X_{r}^{q}+X_{r}^{-\theta}\right) \mathrm{d} r+|\sigma| \mathbb{M}_{B^{H}, T}((t-s)) \\
\leq \tilde{h}\left(1+\|X\|_{s, t, \infty}^{q}+\left\|X^{-1}\right\|_{s, t, \infty}^{\theta}\right)(t-s) \\
\quad+|\sigma| \mathbb{M}_{B^{H}, T}((t-s)),
\end{aligned}
$$

which implies for any $p>0$,

$$
\mathbb{E} \mathbb{M}_{X, T}(h)^{p} \leq C_{T, p}\left(1+\mathbb{E}\|X\|_{0, T, \infty}^{p q}+\mathbb{E}\left\|X^{-1}\right\|_{0, T, \infty}^{\theta p}\right) h^{p}+C_{p}|\sigma|^{p} \mathbb{E}\left(\mathbb{M}_{B^{H}, T}(h)\right)^{p} .
$$

It follows from $\alpha>1$, Lemma 3.3 and the modulus of continuity of $B^{H}$ (see e.g. [26, Theorem 4.2] or [20, Theorem 6.3.3]) that

$$
\mathbb{E M}_{X, T}(h)^{p} \leq C_{p, T}\left\{h^{p}+h^{p H}\left(\log \left(1+\frac{1}{h}\right)\right)^{\frac{p}{2}}\right\} .
$$

By the Hölder inequality and the following inequality

$$
\sup _{|t-s| \leq h, s, t \leq T}\left|X_{t}^{-1}-X_{s}^{-1}\right| \leq \sup _{0 \leq s, t \leq T}\left(\frac{1}{X_{t} X_{s}}\right) \sup _{|t-s| \leq h, s, t \leq T}\left|X_{t}-X_{s}\right|
$$




$$
\leq\left(\sup _{0 \leq t \leq T} X_{t}^{-2}\right) \mathbb{M}_{X, T}(h),
$$

we get the moment estimate of the modulus of continuity of $X^{-1}$.

For $\alpha=1$, one can repeat the argument for $\alpha>1$, and note that negative power moments in (1) of Lemma 3.3 hold for small $T$ depending on $p$.

\section{Numerical Approximation}

In this section, we shall consider the numerical approximation of the following equation

$$
\mathrm{d} X_{t}=b\left(X_{t}\right) \mathrm{d} t+\sigma \mathrm{d} B_{t}^{H}, X_{0}>0 .
$$

The drift term $b(\cdot)$ satisfies (A1), (A2') and (A3'), and all these conditions are independent of time. To ensure the positivity of the numerical scheme, we shall use the backward Euler method as in [15]. Moments estimates obtained in the previous section will be used here.

Let $T>0, N \in \mathbb{N}$ such that $h:=\frac{T}{N}<\left(h_{3} \vee K\right)^{-1}, t_{n}=n h$, and let $\Delta B_{n+1}^{H}=$ $B_{t_{n+1}}^{H}-B_{t_{n}}^{H}$. We introduce the backward Euler scheme. Define $Y_{0}=X_{0}>0$, and consider

$$
Y_{n+1}=Y_{n}+b\left(Y_{n+1}\right) h+\sigma \Delta B_{n+1}^{H}, n \in \mathbb{N} \cup\{0\} .
$$

The equation (4.2) has a unique positive solution $Y_{n+1}, n \geq 0$. To prove this, we consider the following function

$$
U(x)=b(x) h-x, x>0 .
$$

By (A2) and $h>0, \lim _{x \rightarrow 0^{+}} U(x)=+\infty$. By (A2') and $h_{3} h<1$,

$$
\varlimsup_{x \rightarrow+\infty} U(x) \leq \lim _{x \rightarrow+\infty}\left(\left(h_{3} h-1\right) x\right)=-\infty .
$$

Moreover, by (A1) and $h K<1$, we have

$$
U^{\prime}(x)=b^{\prime}(x) h-1 \leq K h-1<0 .
$$

Then for any $c \in \mathbb{R}$, there exists unique $x$ such that $U(x)=c$. Hence, there exists $Y_{n+1}$ such that

$$
U\left(Y_{n+1}\right)=b\left(Y_{n+1}\right) h-Y_{n+1}=-Y_{n}-\sigma \Delta B_{n+1}^{H},
$$

which is equivalent to (4.2). Let

$$
Y_{t}^{h}=\frac{t_{n+1}-t}{t_{n+1}-t_{n}} Y_{n}+\frac{t-t_{n}}{t_{n+1}-t_{n}} Y_{n+1}, t_{n} \leq t \leq t_{n+1}
$$

For a random variable $\xi$, we denote $\|\xi\|_{p}=\left(\mathbb{E}|\xi|^{p}\right)^{\frac{1}{p}}$. In addition to (A1), (A2') and (A3'), we shall impose the following assumptions. 
(H1) The drift term $b \in C^{2}(\mathbb{R})$, and there are nonnegative constants $p_{1}, p_{2}$ and $C>0$ such that

$$
\left|b^{\prime}(x)\right|+\left|b^{\prime \prime}(x)\right| \leq C\left(1+x^{p_{1}}+x^{-p_{2}}\right), x>0 .
$$

Our result on numerical approximation of (4.1) reads as follows.

Theorem 4.1. Assume (A1), (A2'), (A3') and (H1) hold. Let $h_{0}=\left(h_{3} \vee\right.$ $K)^{-1}$ and $h<h_{0}$.

(1) If $\alpha>1$, then

$$
\begin{aligned}
& \mathbb{E} \sup _{0 \leq n \leq N-1}\left|X_{t_{n+1}}-Y_{n+1}\right|^{p} \leq C_{T, X_{0}, \theta, H, p, B} h^{p H}, \\
& \mathbb{E} \sup _{t \in[0, T]}\left|X_{t}-Y_{t}^{h}\right|^{p} \leq C_{T, X_{0}, \theta, H, p, B} h^{p H}\left(\log \left(1+\frac{1}{h}\right)\right)^{p / 2} .
\end{aligned}
$$

(2) If $\alpha=1$, then for $p>0$, there is $T>0$ such that (4.4) and (4.5) hold.

Proof. We only prove the claim for $\alpha>1$. For $\alpha=1$, the negative power moments estimates hold for $T$ depending on the given $p>0$ (see Lemma 3.3). Then for $T$ small enough, the arguments for $\alpha>1$ work well in the small interval, and the claim then can be obtained.

(1) We first prove (4.4). It follows from the definition of $Y_{n+1}$ and the mean value theorem that that

$$
\begin{aligned}
X_{t_{n+1}}-Y_{n+1}= & X_{t_{n}}-Y_{n}+\int_{t_{n}}^{t_{n+1}} b\left(X_{s}\right) \mathrm{d} s-b\left(Y_{n+1}\right) h \\
= & X_{t_{n}}-Y_{n}+\left(b\left(X_{t_{n+1}}\right)-b\left(Y_{n+1}\right)\right) h \\
& \quad-\int_{t_{n}}^{t_{n+1}}\left(b\left(X_{t_{n+1}}\right)-b\left(X_{s}\right)\right) \mathrm{d} s \\
= & X_{t_{n}}-Y_{n}+b^{\prime}\left(Y_{n+1}+\xi_{n+1}\left(X_{t_{n+1}}-Y_{n+1}\right)\right) h\left(X_{t_{n+1}}-Y_{n+1}\right) \\
& \quad-\int_{t_{n}}^{t_{n+1}}\left(\int_{s}^{t_{n+1}} b^{\prime}\left(X_{r}\right) b\left(X_{r}\right) \mathrm{d} r+\sigma \int_{s}^{t_{n+1}} b^{\prime}\left(X_{r}\right) \mathrm{d} B_{r}^{H}\right) \mathrm{d} s
\end{aligned}
$$

where $\xi_{n+1} \in(0,1)$. By (A1) and letting

$$
\Delta_{n+1}=b^{\prime}\left(Y_{n+1}+\xi_{n+1}\left(X_{t_{n+1}}-Y_{n+1}\right)\right),
$$

we have

$$
1-\Delta_{n+1} h \geq 1-K h>0
$$

holds for $h<h_{0}$. On the other hand, it follows from the Fubini theorem that

$$
\int_{t_{n}}^{t_{n+1}}\left(\int_{s}^{t_{n+1}} b^{\prime}\left(X_{r}\right) b\left(X_{r}\right) \mathrm{d} r+\sigma \int_{s}^{t_{n+1}} b^{\prime}\left(X_{r}\right) \mathrm{d} B_{r}^{H}\right) \mathrm{d} s
$$




$$
=\int_{t_{n}}^{t_{n+1}}\left(r-t_{n}\right) b^{\prime}\left(X_{r}\right) b\left(X_{r}\right) \mathrm{d} r+\sigma \int_{t_{n}}^{t_{n+1}}\left(r-t_{n}\right) b^{\prime}\left(X_{r}\right) \mathrm{d} B_{r}^{H} .
$$

Substituting this into (4.6), letting $\Upsilon_{n+1}=X_{t_{n+1}}-Y_{n+1}$ and

$$
Q_{n+1}=-\int_{t_{n}}^{t_{n+1}}\left(r-t_{n}\right) b^{\prime}\left(X_{r}\right) b\left(X_{r}\right) \mathrm{d} r-\sigma \int_{t_{n}}^{t_{n+1}}\left(r-t_{n}\right) b^{\prime}\left(X_{r}\right) \mathrm{d} B_{r}^{H},
$$

we get that

$$
\Upsilon_{n+1}=\left(1-\Delta_{n+1} h\right)^{-1} \Upsilon_{n}+\left(1-\Delta_{n+1} h\right)^{-1} Q_{n+1}
$$

Consequently,

$$
\Upsilon_{n+1}=\sum_{i=1}^{n+1} Q_{i} \prod_{k=i}^{n+1}\left(1-\Delta_{k} h\right)^{-1}=: \sum_{i=1}^{n+1} Q_{i} \rho_{i}
$$

Next, we shall estimate the right hand side of the above equality. Since

$$
\prod_{k=i}^{n+1}\left(1-\Delta_{k} h\right)^{-1} \leq(1-K h)^{-n+i-2} \leq e^{(n+1) \log \frac{1}{1-K h}} \leq e^{\frac{(n+1) K h}{1-K h}}=e^{\frac{K T}{1-K h}}
$$

it follows from (4.7) that

$$
\mathbb{E} \sup _{1 \leq n \leq N}\left|\Upsilon_{n}\right|^{p} \leq C_{T, K} \mathbb{E}\left(\sum_{i=1}^{N}\left|Q_{i}\right|\right)^{p}
$$

By the definition of $Q_{i}$, there are two integrals to be estimated. For the ordinary integral, it follows from (A2'), (A3') and (H1) that

$$
\begin{aligned}
\left\|\sum_{i=1}^{N} \int_{t_{i-1}}^{t_{i}}\left(t_{i-1}-r\right) b^{\prime}\left(X_{r}\right) b\left(X_{r}\right) \mathrm{d} r\right\|_{p} & \leq C_{K, T} h \sum_{i=1}^{N} \int_{t_{i-1}}^{t_{i}}\left\|b^{\prime}\left(X_{r}\right) b\left(X_{r}\right)\right\|_{p} \mathrm{~d} r \\
& \leq C_{T} h \int_{0}^{T}\left\|1+X_{r}^{-\left(\theta+p_{1}\right)}+X_{r}^{q \vee\left(1+p_{2}\right)}\right\|_{p} \mathrm{~d} r \\
& \leq C_{T, \theta, X_{0}, p_{1}, p_{2}, q, K} h .
\end{aligned}
$$

For the stochastic integration, by Proposition 2.2 or [23, Theorem 5.2.3]

$$
\begin{aligned}
& \sum_{i=1}^{N}\left|\int_{t_{i-1}}^{t_{i}}\left(r-t_{i-1}\right) b^{\prime}\left(X_{r}\right) \mathrm{d} B_{r}^{H}\right| \\
& \quad \leq \sum_{i=1}^{N}\left|\int_{0}^{T}\left(t-t_{i-1}\right) b^{\prime}\left(X_{t}\right) \mathbb{1}_{\left(t_{i-1}, t_{i}\right]} \delta B_{t}^{H}\right| \\
& \quad+\sum_{i=1}^{N}\left|\int_{0}^{T} \int_{0}^{T}\left(r-t_{i-1}\right) b^{\prime \prime}\left(X_{r}\right) D_{s} X_{r}\right| s-\left.r\right|^{2 H-2} \mathbb{1}_{\left(t_{i-1}, t_{i}\right]}(r) \mathbb{1}_{(0, r]}(s) \mathrm{d} s \mathrm{~d} r \mid
\end{aligned}
$$




$$
\begin{aligned}
= & \sum_{i=1}^{N}\left|\int_{0}^{T}\left(r-t_{i-1}\right) b^{\prime}\left(X_{r}\right) \mathbb{1}_{\left(t_{i-1}, t_{i}\right]}(r) \delta B_{r}^{H}\right| \\
& +\sum_{i=1}^{N} \int_{t_{i-1}}^{t_{i}} \int_{0}^{r}\left(r-t_{i-1}\right)\left|b^{\prime \prime}\left(X_{r}\right)\right|\left|D_{s} X_{r}\right||s-r|^{2 H-2} \mathrm{~d} s \mathrm{~d} r \\
= & : I_{1}+I_{2} .
\end{aligned}
$$

For $I_{2}$, it follows from (4.3) that

$$
\begin{aligned}
\left\|I_{2}\right\|_{p} & \leq\left\|\sum_{i=1}^{N} \int_{t_{i-1}}^{t_{i}} \int_{0}^{r}\left(r-t_{i-1}\right)\left|b^{\prime \prime}\left(X_{r}\right)\left\|D_{s} X_{r}\right\| s-r\right|^{2 H-2} \mathrm{~d} s \mathrm{~d} r\right\|_{p} \\
& \leq C h \sum_{i=1}^{N} \int_{t_{i-1}}^{t_{i}} \int_{0}^{r}\left\|X_{r}^{-p_{1}}+X_{r}^{p_{2}}\right\|_{p} e^{\int_{s}^{r} K \mathrm{~d} u}|s-r|^{2 H-2} \mathrm{~d} s \mathrm{~d} r \\
& \leq C_{T, K, H} h \sum_{i=1}^{N} \int_{t_{i-1}}^{t_{i}}\left\|X_{r}^{-p_{1}}+X_{r}^{p_{2}}\right\|_{p} r^{2 H-1} \mathrm{~d} s \mathrm{~d} r \\
& \leq C_{T, \theta, H, X_{0}, K} h .
\end{aligned}
$$

For $I_{1}$, it follows from Minkowski's inequality that

$$
\begin{aligned}
\left\|I_{1}\right\|_{p} & =\left\|\sum_{i=1}^{N}\left|\int_{0}^{T}\left(r-t_{i-1}\right) b^{\prime}\left(X_{r}\right) \mathbb{1}_{\left(t_{i-1}, t_{i}\right]} \delta B_{r}^{H}\right|\right\|_{p} \\
& \leq \sum_{i=1}^{N}\left\|\int_{0}^{T}\left(r-t_{i-1}\right) b^{\prime}\left(X_{r}\right) \mathbb{1}_{\left(t_{i-1}, t_{i}\right.} \delta B_{r}^{H}\right\|_{p} .
\end{aligned}
$$

By [23, Proposition 1.5.8],

$$
\begin{aligned}
& \mathbb{E}\left|\int_{0}^{T}\left(r-t_{i-1}\right) b^{\prime}\left(X_{r}\right) \mathbb{1}_{\left(t_{i-1}, t_{i}\right]} \delta B_{r}^{H}\right|^{p} \\
& \leq C_{p}\left(\int_{\left(t_{i-1}, t_{i}\right]^{2}}\left(r-t_{i-1}\right)\left(s-t_{i-1}\right)\left|\mathbb{E} b^{\prime}\left(X_{r}\right)\right|\left|\mathbb{E} b^{\prime}\left(X_{s}\right)\right||r-s|^{2 H-2} \mathrm{~d} r \mathrm{~d} s\right)^{\frac{p}{2}} \\
& \quad+\mathbb{E}\left(\int_{t_{i-1}}^{t_{i}} \int_{t_{i-1}}^{t_{i}} \int_{0}^{r} \int_{0}^{s}\left(r-t_{i-1}\right)\left(s-t_{i-1}\right)\left|b^{\prime \prime}\left(X_{r}\right)\right|\left|b^{\prime \prime}\left(X_{s}\right)\right|\right. \\
& \left.\quad \times\left|D_{u} X_{r}\right|\left|D_{v} X_{s}\right||u-v|^{2 H-2}|s-r|^{2 H-2} \mathrm{~d} u \mathrm{~d} v \mathrm{~d} s \mathrm{~d} r\right)^{\frac{p}{2}} \\
& \leq C_{T, p_{1}, p_{2}, K} h^{p}\left(\int_{\left(t_{i-1}, t_{i}\right]^{2}}|r-s|^{2 H-2} \mathrm{~d} r \mathrm{~d} s\right)^{\frac{p}{2}} \\
& \quad+C_{T, K, H} h^{p}\left(\int_{\left(t_{i-1}, t_{i}\right]^{2}} \int_{0}^{r} \int_{0}^{s}\left(\mathbb{E}\left(1+X_{r}^{-p_{1}}+X_{r}^{p_{2}}\right)^{\frac{p}{2}}\left(1+X_{s}^{-p_{1}}+X_{s}^{p_{2}}\right)^{\frac{p}{2}}\right)^{\frac{2}{p}}\right. \\
& \left.\quad \times|u-v|^{2 H-2}|r-s|^{2 H-2} \mathrm{~d} u \mathrm{~d} v \mathrm{~d} r \mathrm{~d} s\right)^{\frac{p}{2}}
\end{aligned}
$$




$$
\begin{aligned}
& \leq C_{T, X_{0}, H, K} h^{p+H p} \\
& \quad+C_{T, X_{0}, K, H, p_{1}, p_{2}, p} h^{p}\left(\int_{\left(t_{i-1}, t_{i}\right]^{2}} \int_{(0, T]^{2}}|u-v|^{2 H-2}|r-s|^{2 H-2} \mathrm{~d} u \mathrm{~d} v \mathrm{~d} r \mathrm{~d} s\right)^{\frac{p}{2}} \\
& \leq C_{T, X_{0}, K, H, p_{1}, p_{2}, p} h^{p+p H} .
\end{aligned}
$$

Thus

$$
\left\|I_{1}\right\|_{p} \leq C_{T, X_{0}, p, H, K} \sum_{i=1}^{N} h^{1+H} \leq C_{T, X_{0}, p, H, K} h^{H} .
$$

Substituting (4.9), (4.10), (4.11) and (4.12) into (4.8), we obtain

$$
\mathbb{E} \sup _{0 \leq n \leq N-1}\left|\Upsilon_{n+1}\right|^{p} \leq C_{T, X_{0}, \theta, H, p, q, K, p_{1}, p_{2}} h^{p H} .
$$

(2) For $t \in\left[t_{n}, t_{n+1}\right]$,

$$
\begin{aligned}
\left|X_{t}-Y_{t}^{h}\right|=\mid & -\frac{t-t_{n}}{h} X_{t_{n+1}}-\frac{t_{n+1}-t}{h} X_{t_{n}}+X_{t}+\frac{t-t_{n}}{h}\left(X_{t_{n+1}}-Y_{n+1}\right) \\
& \quad+\frac{t_{n+1}-t}{h}\left(X_{t_{n}}-Y_{n}\right) \mid \\
\leq & \left|X_{t_{n+1}}-X_{t}\right|+\left|X_{t_{n}}-X_{t}\right|+\left|\Upsilon_{n+1}\right|+\left|\Upsilon_{n}\right| \\
\leq & 2 \int_{t_{n}}^{t_{n+1}}\left|b\left(X_{r}\right)\right| \mathrm{d} r+\left|B_{t_{n+1}}^{H}-B_{t}^{H}\right|+\left|B_{t}^{H}-B_{t_{n}}^{H}\right| \\
& \quad+\left|\Upsilon_{n+1}\right|+\left|\Upsilon_{n}\right| \\
\leq & C \int_{t_{n}}^{t_{n+1}}\left(1+X_{r}^{q \vee 1}+X_{r}^{-\theta}\right) \mathrm{d} r+2 \mathbb{M}_{H, T}(h)+\left|\Upsilon_{n+1}\right|+\left|\Upsilon_{n}\right| \\
\leq & C\left(\left(1+\|X\|_{0, T, \infty}^{q \vee 1}\right) h+\left(\int_{0}^{T} X_{r}^{-\frac{\theta}{1-H}} \mathrm{~d} r\right)^{1-H} h^{H}\right) \\
& \quad+2 \mathbb{M}_{B^{H}, T}(h)+2 \sup _{1 \leq n \leq N}\left|\Upsilon_{n}\right| \\
= & I_{1}+I_{2}+I_{3} .
\end{aligned}
$$

Since $I_{1}, I_{2}$ and $I_{3}$ are independent of $n$ and $t$, we have

$$
\mathbb{E} \sup _{t \in[0, T]}\left|X_{t}-Y_{t}^{h}\right|^{p} \leq 3^{p-1}\left(\mathbb{E} I_{1}^{p}+\mathbb{E} I_{2}^{p}+\mathbb{E} I_{3}^{p}\right) .
$$

It follows from Lemma 3.3 and Lemma 3.4 that

$$
\mathbb{E} I_{1}^{p} \leq C_{K, q, T, X_{0}, p, \theta, H} h^{p H} .
$$

The inequality (4.4) yields that

$$
\mathbb{E} I_{3}^{p}=2^{p} \mathbb{E} \sup _{1 \leq n \leq N}\left|\Upsilon_{n}\right|^{p} \leq C_{K, q, T, X_{0}, p, \theta, H} h^{p H} .
$$


The modulus of continuity of $B^{H}$ (see e.g. [26, Theorem 4.2] or [20, Theorem 6.3.3]) implies that there is a constant $C_{T, p}>0$ such that

$$
\mathbb{E} I_{2}^{p}=2^{p} \mathbb{E M}_{B^{H}, T}^{p}(h) \leq C_{T, p} h^{p H}(\log (1+1 / h))^{p / 2},
$$

Hence,

$$
\mathbb{E} \sup _{t \in[0, T]}\left|X_{t}-Y_{t}^{h}\right|^{p} \leq C_{K, q, T, X_{0}, p, \theta, H} h^{p H}(\log (1+1 / h))^{p / 2} .
$$

The proof is therefore complete.

Before we provide examples to illustrate Theorem 4.1, we need following corollary for future use.

Corollary 4.2. Assume the conditions of Theorem 4.1 hold.

(1) If $\alpha>1$, then for any $l>0$,

$$
\left(\mathbb{E} \sup _{t \in[0, T]}\left|X_{t}^{l}-\left(Y_{t}^{h}\right)^{l}\right|^{p}\right)^{\frac{1}{p}} \leq C h^{H(l \wedge 1)}(\log (1+1 / h))^{\frac{l \wedge 1}{2}}
$$

for any $l \in(0, \alpha]$,

$$
\left(\mathbb{E} \sup _{t \in[0, T]}\left|X_{t}^{-l}-\left(Y_{t}^{h}\right)^{-l}\right|^{p}\right)^{\frac{1}{p}} \leq C_{p, T} h^{(2 H-1)(l \wedge 1)}(\log (1+1 / h))^{l \wedge 1}
$$

(2) If $\alpha=1$, then for $l>0$ and $p>0$, there is $T>0$ such that (4.13) holds; for $l \in(0,1]$ and $p>0$, there is $T>0$ such that (4.14) holds.

Proof. For $l \in(0,1]$, it follows from Lemma 4.1, the basic inequality

$$
\left|a^{l}-b^{l}\right| \leq|a-b|^{l}, \quad a>0, b>0,
$$

and Jessen's inequality that

$$
\begin{aligned}
\left(\mathbb{E} \sup _{t \in[0, T]}\left|X_{t}^{l}-\left(Y_{t}^{h}\right)^{l}\right|^{p}\right)^{\frac{1}{p}} & \leq\left(\mathbb{E} \sup _{t \in[0, T]}\left|X_{t}-Y_{t}^{h}\right|^{l p}\right)^{\frac{1}{p}} \leq\left(\mathbb{E} \sup _{t \in[0, T]}\left|X_{t}-Y_{t}^{h}\right|^{p}\right)^{\frac{l}{p}} \\
& \leq C h^{l H}\left(\log \left(1+\frac{1}{h}\right)\right)^{\frac{l}{2}}
\end{aligned}
$$

For $l>1$,

$$
\begin{aligned}
& \left(\mathbb{E} \sup _{t \in[0, T]}\left|X_{t}^{l}-\left(Y_{t}^{h}\right)^{l}\right|^{p}\right)^{\frac{1}{p}} \\
& \quad \leq\left(\mathbb{E} \sup _{t \in[0, T]}\left(X_{t}^{p(l-1)} \vee\left(Y_{t}^{h}\right)^{p(l-1)}\right)\left|X_{t}-Y_{t}^{h}\right|^{p}\right)^{\frac{1}{p}}
\end{aligned}
$$




$$
\begin{aligned}
& \leq\left(\mathbb{E} \sup _{t \in[0, T]}\left(X_{t}^{2 p(l-1)} \vee\left(Y_{t}^{h}\right)^{2 p(l-1)}\right)\right)^{\frac{1}{2 p}}\left(\mathbb{E} \sup _{t \in[0, T]}\left|X_{t}-Y_{t}^{h}\right|^{2 p}\right)^{\frac{1}{2 p}} \\
& \leq C_{p, T} h^{H}\left(\log \left(1+\frac{1}{h}\right)\right)^{\frac{1}{2}} .
\end{aligned}
$$

Hence, we have proved our first claim.

To consider the negative power approximation, we first give an estimate of $Y_{n}^{-1}$. By (4.2), (A2) and (A3'), there is positive constant $C$ which is independent of $n, h$ such that

$$
\begin{aligned}
& C\left(Y_{n+1}^{-\alpha} h-\left(Y_{n+1}^{q}+1\right) h\right) \leq b\left(Y_{n+1}\right) h \\
& \quad \leq\left|Y_{n+1}-X_{t_{n+1}}\right|+\left|X_{t_{n+1}}-X_{t_{n}}\right|+\left|Y_{n}-X_{t_{n}}\right|+|\sigma|\left|B_{n+1}^{H}\right| .
\end{aligned}
$$

Then

$$
\begin{aligned}
C \sup _{1 \leq n \leq N} Y_{n}^{-\alpha} \leq & \sup _{1 \leq n \leq N}\left(Y_{n}^{q}+1\right)+\frac{2}{h} \sup _{1 \leq n \leq N}\left|Y_{n}-X_{t_{n}}\right| \\
& +\frac{1}{h} \sup _{1 \leq n \leq N}\left|X_{t_{n+1}}-X_{t_{n}}\right|+\frac{|\sigma|}{h} \mathbb{M}_{B^{H}, T}^{p}(h) \\
\leq & C \sup _{1 \leq n \leq N}\left(Y_{n}^{q}+1\right)+\frac{2}{h} \sup _{1 \leq n \leq N}\left|Y_{n}-X_{t_{n}}\right| \\
& +\frac{1}{h} \mathbb{M}_{X, T}(h)+\frac{|\sigma|}{h} \mathbb{M}_{B^{H}, T}^{p}(h) .
\end{aligned}
$$

By (4.4), it is clear that for all $p>0$, we have $\mathbb{E} \sup _{1 \leq n \leq N}\left(Y_{n}^{q p}\right)<\infty$. Due to Theorem 4.1,

$$
\mathbb{E} \sup _{1 \leq n \leq N}\left|Y_{n}-X_{t_{n}}\right|^{p} \leq h^{H p}\left(\log \left(1+\frac{1}{h}\right)\right)^{\frac{p}{2}} .
$$

It follows from Lemma 3.4 that

$$
\mathbb{E M}_{X, T}^{p}(h) \leq h^{H p}\left(\log \left(1+\frac{1}{h}\right)\right)^{\frac{p}{2}} .
$$

Combining these with (4.15), we obtain

$$
\mathbb{E} \sup _{1 \leq n \leq N} Y_{n}^{-p \alpha} \leq C\left(1+h^{(H-1) p}\left(\log \left(1+\frac{1}{h}\right)\right)^{\frac{p}{2}}\right) .
$$

Then for $l \in[1, \alpha]$, it follows from (4.16) that

$$
\begin{aligned}
& \left(\mathbb{E} \sup _{t \in[0, T]}\left|X_{t}^{-l}-\left(Y_{t}^{h}\right)^{-l}\right|^{p}\right)^{\frac{1}{p}}=\left(\mathbb{E} \sup _{t \in[0, T]} \frac{\left|X_{t}^{l}-\left(Y_{t}^{h}\right)^{l}\right|^{p}}{X_{t}^{p l}\left(Y_{t}^{h}\right)^{p l}}\right)^{\frac{1}{p}} \\
& \quad \leq\left(\mathbb{E} \sup _{t \in[0, T]} X_{t}^{-3 p l}\right)^{\frac{1}{3 p}}\left(\mathbb{E} \sup _{t \in[0, T]}\left(Y_{t}^{h}\right)^{-3 p l}\right)^{\frac{1}{3 p}}\left(\mathbb{E} \sup _{t \in[0, T]}\left|X_{t}^{l}-\left(Y_{t}^{h}\right)^{l}\right|^{3 p}\right)^{\frac{1}{3 p}}
\end{aligned}
$$




$$
\begin{aligned}
& =\left(\mathbb{E} \sup _{t \in[0, T]} X_{t}^{-3 p l}\right)^{\frac{1}{3 p}}\left(\mathbb{E} \sup _{1 \leq n \leq N} Y_{n}^{-3 p l}\right)^{\frac{1}{3 p}}\left(\mathbb{E} \sup _{t \in[0, T]}\left|X_{t}^{l}-\left(Y_{t}^{h}\right)^{l}\right|^{3 p}\right)^{\frac{1}{3 p}} \\
& \leq C_{p, T}\left(1+h^{H-1}\left(\log \left(1+\frac{1}{h}\right)\right)^{\frac{1}{2}}\right) h^{H}\left(\log \left(1+\frac{1}{h}\right)\right)^{\frac{1}{2}} \\
& \leq C_{p, T} h^{2 H-1} \log \left(1+\frac{1}{h}\right) .
\end{aligned}
$$

For $l<1$,

$$
\begin{aligned}
\left(\mathbb{E} \sup _{t \in[0, T]}\left|X_{t}^{-l}-\left(Y_{t}^{h}\right)^{-l}\right|^{p}\right)^{\frac{1}{p}} & \leq\left(\mathbb{E} \sup _{t \in[0, T]}\left|X_{t}^{-1}-\left(Y_{t}^{h}\right)^{-1}\right|^{p}\right)^{\frac{l}{p}} \\
& \leq C_{p, T, l} h^{(2 H-1) l}\left(\log \left(1+\frac{1}{h}\right)\right)^{l} .
\end{aligned}
$$

Combining these two cases together, we prove our second conclusion.

Remark 4.1. If $\phi$ is a continuous function on $(0, \infty)$ such that

$$
|\phi(x)-\phi(y)| \leq C\left|x^{l}-y^{l}\right| \quad \text { or } \quad|\phi(x)-\phi(y)| \leq C\left|x^{-l}-y^{-l}\right|
$$

for $l$ as in Corollary 4.2 and some $C>0$, then we can approximate $\phi\left(X_{t}\right)$ by $\phi\left(Y_{t}^{h}\right)$.

For $\alpha=1$, the convergence of the backward Euler scheme for $C$-I-R model driven by fractional Brownian motion has been obtained in [15]. Theorem 4.1 and Corollary 4.2 in our paper can also be applied to $C$-I- $R$ model.

Finally, we apply our results to the two examples introduced in the introduction.

Example 4.1. We consider the numerical simulation of the following equation

$$
\mathrm{d} Z_{t}=\left(a_{1}-a_{2} Z_{t}\right) \mathrm{d} t+\sigma Z_{t}^{\gamma} \mathrm{d} B_{t}^{H}, Z_{0}>0
$$

with $\gamma \in\left(\frac{1}{2}, 1\right), a_{1}>0, a_{2} \in \mathbb{R}$ and $\sigma \neq 0$. To study this equation, we consider

$$
\mathrm{d} X_{t}=(1-\gamma)\left(a_{1} X_{t}^{-\frac{\gamma}{1-\gamma}}-a_{2} X_{t}\right) \mathrm{d} t+\sigma(1-\gamma) \mathrm{d} B_{t}^{H}, X_{0}=Z_{0}^{1-\gamma} .
$$

Setting $b(x)=(1-\gamma) a_{1} x^{-\frac{\gamma}{1-\gamma}}-a_{2}(1-\gamma) x$, it is clear that (A1), (A2') and (A3') hold with $K=a_{2}^{-}, \theta=\alpha=-\frac{\gamma}{1-\gamma}$, and $q=\mathbb{1}_{\left[a_{2}>0\right]}$. Then this equation has a unique solution by applying Theorem 3.1. Moreover, it follows from the chain rule that $Z_{t}=X_{t}^{\frac{1}{1-\gamma}}$ and (4.17) has a unique solution. Let $Y_{t}^{h}$ be the numerical solution of $X_{t}$ and $h_{0}=\left(\left[(1-\gamma) a_{1}\right] \vee a_{2}^{-}\right)^{-1}$. It follows from Corollary 4.2 that

$$
\left(\mathbb{E} \sup _{t \in[0, T]}\left|Z_{t}-\left(Y_{t}^{h}\right)^{\frac{1}{1-\gamma}}\right|^{p}\right)^{\frac{1}{p}} \leq C h^{H}(\log (1+1 / h))^{\frac{1}{2}}, h<h_{0} .
$$


Example 4.2. In this example, we investigate the nonlinear Aït-Sahalia-type interest rate model:

$$
\mathrm{d} Z_{t}=\left(a_{-1} Z_{t}^{-1}-a_{0}+a_{1} Z_{t}-a_{2} Z_{t}^{r}\right) \mathrm{d} t+\sigma Z_{t}^{\rho} \mathrm{d} B_{t}^{H}, Z_{0}>0
$$

with $r+1>2 \rho$ and $r \geq 2 \wedge \rho+1>2$ and $a_{i}>0, i=-1,0,1,2$. To investigate the numerical solutions of (4.18), we consider

$$
\begin{aligned}
\mathrm{d} X_{t}=( & \rho-1)\left(a_{2} X_{t}^{-\frac{r-\rho}{\rho-1}}-a_{1} X_{t}+a_{0} X_{t}^{\frac{\rho}{\rho-1}}-a_{-1} X_{t}^{\frac{\rho+1}{\rho-1}}\right) \mathrm{d} t \\
& +(1-\rho) \sigma \mathrm{d} B_{t}^{H}, X_{0}=Z_{0}^{1-\rho} .
\end{aligned}
$$

Set

$$
\begin{aligned}
b(x) & =(\rho-1)\left(a_{2} x^{-\frac{r-\rho}{\rho-1}}-a_{1} x+a_{0} x^{\frac{\rho}{\rho-1}}-a_{-1} x^{\frac{\rho+1}{\rho-1}}\right) \\
& \equiv b_{1} x^{-\frac{r-\rho}{\rho-1}}-b_{2} x+b_{3} x^{\frac{\rho}{\rho-1}}-b_{4} x^{\frac{\rho+1}{\rho-1}} .
\end{aligned}
$$

Since $\frac{r-\rho}{\rho-1}>1$, it is clear that (A1), (A2') and (A3') hold with $\theta=\alpha=\frac{r-\rho}{\rho-1}$, $q=\frac{\rho+1}{\rho-1}$ and some constant $K$. Then this equation has a unique solution, and so does (4.18). Moreover $Z_{t}=X_{t}^{-\frac{1}{\rho-1}}$. It is clear by $\frac{r-\rho}{\rho-1}>1$ and $\frac{\rho+1}{\rho-1}>\frac{\rho}{\rho-1}$ that for $h>0$

$$
\lim _{x \rightarrow 0^{+}}(b(x) h-x)=+\infty, \quad \lim _{x \rightarrow+\infty}(b(x) h-x)=-\infty .
$$

On the other hand,

$$
\begin{aligned}
b^{\prime}(x) h-1=- & \frac{b_{1}(r-\rho) h}{\rho-1} x^{-\frac{r+1}{\rho-1}}-\left(b_{2} h+1\right) \\
& +\frac{b_{3} \rho h}{\rho-1} x^{\frac{1}{\rho-1}}-\frac{b_{4}(\rho+1) h}{\rho-1} x^{\frac{2}{\rho-1}} .
\end{aligned}
$$

Then for $0<h<\frac{4(\rho-1) b_{4}(\rho+1)}{b_{3}^{2} \rho^{2}}$, we have

$$
\frac{\left(b_{3} \rho\right)^{2} h^{2}}{(\rho-1)^{2}}-4\left(\frac{b_{1}(r-\rho) h}{\rho-1} x^{\frac{r+1}{\rho-1}}+b_{2} h+1\right) \frac{b_{4}(\rho+1) h}{\rho-1}<0,
$$

which implies that $b^{\prime}(x) h-1<0$. Consequently, (H1) holds. Hence, Theorem 4.1 can be applied to (4.19) for $h<\frac{4(\rho-1) b_{4}(\rho+1)}{b_{3}^{2} \rho^{2}}$.

Let $Y_{t}^{h}$ be the numerical approximation of $X_{t}$, since $r+1>2 \rho$ and $r>$ $2 \wedge \rho+1$, we have $\frac{1}{\rho-1} \leq \frac{r-\rho}{\rho-1}$. Letting $l=\frac{1}{\rho-1}$ in Corollary 4.2, we then have numerical approximation of $Z_{t}$ such that

$$
\left(\mathbb{E} \sup _{t \in[0, T]}\left|Z_{t}-\left(Y_{t}^{h}\right)^{\frac{1}{\rho-1}}\right|^{p}\right)^{\frac{1}{p}} \leq C_{p, T} h^{(2 H-1)\left(\frac{1}{\rho-1} \wedge 1\right)}(\log (1+1 / h))^{\frac{1}{\rho-1} \wedge 1},
$$

which implies that

$$
\lim _{h \rightarrow 0^{+}} \mathbb{E} \sup _{t \in[0, T]}\left|Z_{t}-\left(Y_{t}^{h}\right)^{\frac{1}{\rho-1}}\right|^{p}=0
$$


Acknowledgement The authors would like to thank the associated editor and referees for their helpful comments and suggestions.

\section{References}

[1] Y. Aït-Sahalia. Testing Continuous-Time Models of the Spot Interest Rate. Rev. Financ. Stud. (1996) 9(2), 385-426.

[2] A. Alòs and D. Nualart. Stochastic integration with respect to the fractional Brownian motion, Stoch. Stoch. Rep. (2003) 75, 129-152.

[3] E. Alòs, O. Mazet and D. Nualart, Stochastic calculus with respect to Gaussian processes, Ann. Probab. (2001) 29, 766-801.

[4] R. J. Barton and H. V. Poor. Signal detection in fractional gaussian noise. IEEE trans. Information Theory (1988) 34, 943-959.

[5] A. Berkaoui, M. Bossy and A. Diop. Euler scheme for SDEs with nonLipschitz diffusion coefficient: strong convergence. ESAIM: Proba. and Stat., (2008) 12, 1-11.

[6] F. Biagini, Y. Hu, B. Øksendal, T. Zhang. Stochastic Calculus for Fractional Brownian Motion and Applications. Springer, London, 2008.

[7] D. Brigo, F. Mercurio. Interest Rate Models-Theory and Practice: With Smile, Inflation and Credit. (2nd ed.) Springer Verlag, (2001).

[8] K. C. Chan, G. A. Karolyi, F. Longstaff, and A. Sanders. An Empirical Comparison of Alternative Models of the Short-Term Interest Rate. The Journal of Finance, Vol. XLVII, No. 3 July (1992).

[9] J. C. Cox, J. E. Ingersoll Jr, and S. A. Ross, A theory of term structure of interest rates. Econometrica (1985) 53, 385-407.

[10] L. Decreusefond and A. S. Üstünel, Stochastic analysis of the fractional Brownian motion, Potential Anal. (1999) 10, 177-214.

[11] S. Dereich, A. Neuenkirch and L. Szpruch. An Euler-type method for the strong approximation of the Cox-Ingersoll-Ross process. Proc. R. Soc. A (2012) 468, 1105-1115.

[12] X.-L. Fan and S.-Q. Zhang. On SDEs driven by fractional Brownian motions with irregular drifts. (2018). arXiv:1810.01669v1.

[13] I. Gyöngy and M. Rásonyi. A note on Euler approximations for SDEs with Hölder continuous diffusion coefficients. Stoch. Proc. Appl. (2011) 121, 2189-2200. 
[14] M. Fischer and G. Nappo. On the Moments of the Modulus of Continuity of Itô Processes. Stoch. Anal. Appl. (2010) 28, 2013-1222.

[15] J. Hong, C. Huang, M. Kamrani and X. Wang, Optimal Strong Convergence Rate of a Backward Euler Type Scheme for the Cox-Ingersoll-Ross Model Driven by Fractional Brownian Motion, Stoch. Proc. Appl. (2019). In press, dio:10.1016/j.spa.2019.07.014.

[16] Y. Hu, Y. Liu, and D. Nualart. Crank-Nicolson scheme for stochastic differential equations driven by fractional Brownian motion. arXiv 1709.01614

[17] Y. Hu, Y. Liu, and D. Nualart. Rate of convergence and asymptotic error distribution of Euler approximation schemes for fractional diffusions, Ann. Appl. Probab. (2016) 26, 1147-1207.

[18] Y. Hu, D. Nualart and X. Song. A singular stochastic differential equation driven by fractional Brownian motion. Stat. \& Prab. Letter (2008), 20752085 .

[19] X. Mao, A. Truman and C. Yuan. Euler-Maruyama approximations in mean-reverting stochastic volatility model under regime-switching. International Journal of Stochastic Analysis (2006), 1-20.

[20] M. Marcus and J. Rosen. Markov Processes, Gaussian Processes and Local Times. Cambridge University Press, New York. (2006).

[21] Y. Mishura, A. Yurchenko-Tytarenko. Fractional Cox-Ingersoll-Ross process with non-zero $<<$ mean $>>$. Modern Stochastics: Theory and Applications (2018) 5 (1): 99-111.

[22] A. Neuenkirch. Optimal approximation of SDE's with additive fractional noise. J. Complexity (2006) 22: 459-474.

[23] D. Nualart. The Malliavin Calculus and Related Topics, 2nd Edition. Springer-Verlag Berlin Heidelberg, 2006.

[24] S. Rostek and R. Schöbel. A note on the use of fractional Brownian motion for financial modeling. Economic Modelling, (2013) 30: 30-35.

[25] L. Szpruch, X. Mao, D. J. Higham and J. Pan. Numerical simulation of a strongly nonlinear Ait-Sahalia-type interest rate model, BIT Numer. Math. (2011) 51: 405-425.

[26] Y. Xiao. Sample Path Properties of Anisotropic Gaussian Random Fields, in "A Minicourse on Stochastic Partial Differential Equations", Lecture Notes in Mathematics, 1962, 145-212.

[27] M. Zähle, Integration with respect to fractal functions and stochastic calculus I, Probab. Theory Related Fields (1998) 111, 333-374. 